\title{
Inclusão Digital no Ensino de lluminação Natural
}

\author{
FARIA, João Roberto Gomes de ${ }^{1}$ \\ ${ }^{1}$ Universidade Estadual Paulista, Bauru, Brasil. joaofari@faac.unesp.br
}

\section{Resumo}

As atividades de ensino/aprendizado de iluminação natural do ambiente construído em cursos de Arquitetura e Urbanismo exigem do aluno a capacidade de abstrair os efeitos da luz no dia distribuídos no espaço tridimensional que está em projeto. Diversas ferramentas e técnicas podem ser usadas para facilitar a compreensão dos fenômenos envolvidos, dentre as quais a simulação computacional. Este artigo relata a inclusão digital do ensino de iluminação natural no curso de Arquitetura e Urbanismo da Faculdade de Arquitetura, Artes e Comunicação de Bauru (FAAC) da UNESP - Universidade Estadual Paulista, ocorrida a partir de 2010. Essa inclusão envolveu a utilização de software gratuito, especificamente dos programas DIALux e SketchUp+Radiance, ambos com saídas gráficas para visualização das cenas iluminadas e para análise de resultados. O modelo gráfico é exportado do SketchUp para o Radiance através de um plugin e para a simulação da iluminação foi desenvolvida uma interface amigável para ambiente Microsoft Windows. O processo de inclusão digital está consolidado, com ampla aceitação por parte dos alunos, para os quais a simulação computacional facilita o entendimento da relação entre a luz do dia e o ambiente construído e auxilia no projeto de elementos de controle da iluminação natural.

Palavras-Chave: ensino, conforto visual, modelagem computacional.

\begin{abstract}
The teaching/learning activities of the daylighting built environment require from the Architecture and Urbanism undergraduate student the ability to abstract the effects of daylight distributed in three-dimensional space that is being designed. Several tools and techniques can be used to facilitate the understanding of the involved phenomena, among which the computational simulation. This paper reports the digital inclusion of the daylighting teaching in the Architecture and Urbanism undergraduate course at the School of Architecture, Arts and Social Communication of Bauru (FAAC) of UNESP - Sao Paulo State University, that began in 2010. The inclusion process involved free software use, specifically the programs DIALux and SketchUp+Radiance, both with graphical output for the illuminated scenes visualization and for result analysis. The graphic model is converted from SketchUp to Radiance by a plugin and a user-friendly interface for Windows was developed to simulate the lighting. The process of digital inclusion is consolidated, with wide acceptance by students, for which computational simulation facilitates understanding of relation between daylight and built environment and helps the design process of elements for daylighting control.
\end{abstract}

Keywords: learning, visual comfort, computational modelling. 


\section{Introdução}

Atividades de ensino/aprendizagem da iluminação natural do espaço construído exigem que 0 aluno abstraia, a partir de modelos gráficos, físicos ou digitais - do projeto que está desenvolvendo, os efeitos que a luz do dia the proporcionará. Para explorar as diversas habilidades cognitivas dos alunos, várias técnicas e ferramentas podem ser empregadas, como o desenho de observação (SCARAZZATO et al., 2005), a modelagem física em escala reduzida (PEREIRA; CASTAÑO; ATANASIO, 2007) e os recursos computacionais (SABRY et al., 2010).

A inclusão digital do ensino da iluminação natural em cursos de Arquitetura e Urbanismo responde a demandas diversas, do avanço do estado da arte à demanda das novas gerações de alunos, formadas num novo ambiente tecnológico, marcado pela cultura digital. Ela traz consigo uma série de recursos e possibilidades, do estudo comparativo de prédios locais a obras arquitetônicas distantes, nas suas respectivas condições de céu; da proposição de esboços volumétricos preliminares até projetos finais detalhados, da criação de ambientes tridimensionais virtuais até a geração de modelos físicos, através de impressoras tridimensionais.

Este artigo tem por objetivo descrever e analisar a inclusão de recursos computacionais nas atividades de ensino/aprendizagem da iluminação natural no ambiente construído no curso de Arquitetura e Urbanismo da Faculdade de Arquitetura, Artes e Comunicação (FAAC) da UNESP - Universidade Estadual Paulista, através de programas computacionais gratuitos, num processo iniciado em 2010. A opção pelo software gratuito está incluída na política atual da UNESP da opção pelo software livre que, para além do aspecto econômico da universidade, permite que todos os alunos tenham acesso a ele em seus próprios computadores e gera, para o educador, possibilidades de desenvolvimento de novas ferramentas, como ocorre no caso aqui relatado.

Para o levantamento do estado da arte do uso de recursos computacionais no ensino de iluminação natural em cursos de graduação de Arquitetura e Urbanismo foi realizada uma pesquisa envolvendo o Portal de Periódicos da Capes (<http://www-periodicos-capes-gov-br. ez87. periodicos.capes.gov.br/index.php? option=com_phome $>$ ), os anais online da PLEA (Passive and Low Energy Architecture, <http:// plea-arch.org/?page_id=421>), a Coletânea de anais dos ENCACs (Encontros Nacionais de
Conforto no Ambiente Construído, disponíveis em DVDs), o portal Google Acadêmico (<http:// scholar.google.com.br/ schhp?hl=pt-BR>) e o próprio portal da Google (<http://www.google. com.br>).

Inicialmente vale a pena destacar que, de acordo com Roberts; Marsh (2001), a natureza do processo do projeto arquitetônico é visual, em especial nas fases iniciais da concepção, quando a forma ainda está sendo desenvolvida. Assim, também a modelagem digital deve ocorrer num ambiente comum aos futuros profissionais, através de desenhos, não sob a forma numérica ou de algoritmos; de forma similar, os resultados devem ilustrar o que se espera da iluminação, ou seja, a visualização de cenas iluminadas e não gráficos ou tabelas. Portanto, aparentemente 0 sucesso do uso de um recurso educacional digital está associado, no ensino de conteúdos de Arquitetura e Urbanismo, à familiaridade que sua interface apresente aos alunos.

Várias tentativas de incorporar a simulação computacional ao ensino de iluminação arquitetônica vêm sendo feitas a partir da década de 1990, com a popularização de computadores pessoais e da internet, através de uma série de recursos.

Uma primeira categoria de ferramentas que exploram a interatividade proporcionada pelo computador é a dos chamados ambientes virtuais de aprendizado. Exemplos deles são o Daylight Visual Effects (DVE) de Lee; Hardin (1996), o Virtual Lighting Laboratory (VLL) de Inanici (2003) e o Virtual Learning Environment (VLE) de Atanasio; Pereira; Pereira (2006), entre outros. Algumas dessas plataformas iterativas foram também disponibilizadas via páginas interativas na internet, como o DayMedia, de 1998 (disponível atualmente somente offline em $<$ http://www.new-learn.info/packages/daymedia/ download/daymedia-1.1.0.zip>) e o Daylighting Pattern Guide (disponível em < http://patternguide. advancedbuildings.net/>). Infelizmente não foram encontrados na pesquisa bibliográfica relatos de experiências didáticas com esse tipo de recurso.

Outra categoria é a dos programas de simulação da iluminação, desenvolvidos originalmente para a pesquisa ou para aplicações profissionais.

Os primeiros programas computacionais de simulação da iluminação no ambiente construído datam da década de 1980, tendo como marco o Radiance (WARD, 1994), desenvolvido originalmente no Lawrence Berkeley National Laboratory. A iluminação a partir da luz do dia foi validada por Mardaljevic (1999) e ele tem sido 
usado em um grande número de aplicações, tanto autonomamente como servindo de motor a outros programas, como o DAYSIM (REINHART, 2001). O programa foi desenvolvido como um conjunto de aplicativos (aproximadamente 100) para plataformas Unix, sem interface gráfica própria, mas seus próprios autores criaram posteriormente uma versão dele para operar em plataforma Microsoft Windows em conjunto com o AutoCAD, o Desktop Radiance, que funcionou somente para as versões 14 e 2000 daquele programa. $O$ Radiance tem possibilidade de gerar uma grande quantidade de produtos de saída, dentre as quais, relevantes a este trabalho, imagens para a visualização de cenas iluminadas e para análise de distribuição de iluminâncias e de luminâncias.

Existem também disponíveis gratuitamente dois programas brasileiros de simulação desenvolvidos originalmente para pesquisas: o APOLUX (CLARO; PEREIRA; LEDO, 2005) e o TROPLUX (CABÚS, 2011). O primeiro importa modelos do AutoCAD e o segundo cria seus modelos a partir de um sistema de entrada de dados numéricos tabular. A saída de dados do primeiro é feita através de imagens de cenas iluminadas e de análise, e gráficos de vários tipos, enquanto as do TROPLUX privilegiam os gráficos.

A linha de programas simuladores gratuitos de iluminação para uso profissional é composta por programas voltados originalmente para projetos de iluminação elétrica, como o DIALux (da DIAL $\mathrm{GMBH}$, Alemanha) ou o Relux (da Relux Informatik AG, Suiça). Eles são desenvolvidos em parceria com fornecedores de sistemas de iluminação, cujos produtos compõem os bancos de dados dos programas. Em função das novas diretrizes de iluminação europeias para melhorar a eficiência energética dos projetos, os programas passaram a simular também a iluminação natural, mas no momento contam com possibilidades restritas de opções de tipos de céus. Tanto o DIALux como o Relux têm a seu favor interfaces gráficas de entrada e saída próprias de uso bastante simples, uma série de bibliotecas de acessórios (como mobiliário e materiais de acabamento) e ferramentas de análise. Em compensação, a elaboração de formas geométricas complexas apresenta dificuldades em função das limitações das interfaces gráficas (SHIKDER, 2009).

O levantamento acerca do uso de programas computacionais no ensino de iluminação em cursos de graduação em Arquitetura e Urbanismo revelou um número muito pequeno de experiências relatadas ou de programas de disciplinas dos quais eles constam. Internacionalmente podem ser citados os relatos de Compagnon (1997), na Universidade de Cambridge e na Ecole d'Ingénieurs de Fribourg, e os programas de disciplinas de cursos de Arquitetura ministradas por Christoph Reinhart e outros na McGill School of Architecture de 2005 a 2007 (disponíveis em <http://www.arch.mcgill. ca/prof/reinhart/arch447/>) e no Massachusetts Institute of Technology a partir de 2008, todos eles usando o Radiance. Por esse levantamento, o uso educacional do DIALux e do Relux se restringe à formação de profissionais da área de iluminação e nada foi encontrado sobre o uso educacional do APOLUX e do TROPLUX.

\section{O uso didático de software gratuito de simulação da iluminação no curso de arquitetura e urbanismo da FAAC}

Como ocorre também em outras escolas brasileiras (SCARAZZATO et al., 2005), o ensino de iluminação no curso de Arquitetura e Urbanismo da FAAC-UNESP se dá de forma independente do projeto arquitetônico. Até 2012 ele ocorria no primeiro semestre do segundo ano. Atualmente, em função da reestruturação do projeto político-pedagógico, a disciplina é oferecida no quinto semestre do curso. Nesses períodos do curso, os alunos já tiveram disciplinas com conteúdos relacionados à percepção do espaço, técnicas de representação e expressão e conforto térmico. Os primeiros projetos arquitetônicos são esboçados ao longo dos terceiro e quarto semestre, incorporando, simultaneamente, técnicas de representação (MAGAGNIN, 2012). Nesse contexto, o ensino da iluminação natural incorpora mais uma variável, conferindo ao espaço a sensação de tridimensionalidade proporcionada pela distribuição da luz do dia. O conteúdo de iluminação natural envolve inicialmente a percepção do espaço iluminado, seguida da análise qualitativa da iluminação e, finalmente, o projeto do aproveitamento da luz do dia envolvendo aspectos qualitativos e quantitativos.

Até 2010 as ferramentas de ensino de iluminação natural eram compostas por medições in loco de variáveis fotométricas e respectivo tratamento gráfico, desenhos de observação, modelos físicos e imagens HDR (high dynamic range larga faixa dinâmica) em sua etapa analítica. $\mathrm{Na}$ etapa preditiva eram usados novamente os modelos físicos e os procedimentos da NBR15215-3 (ASSOCIAÇÃO BRASILEIRA DE NORMAS TÉCNICAS, 2007). 
O histórico da utilização de programas de simulação da iluminação na FAAC-UNESP se iniciou em 2000, com o oferecimento da disciplina optativa Projeto de lluminação para alunos dos dois últimos anos do curso de Arquitetura e Urbanismo. Nela, o programa auxilia o detalhamento do projeto, além de fornecer relatórios complementares e imagens de apresentação. As pranchas do projeto final, infelizmente, ainda precisam ser desenhadas à parte, uma vez que as fornecidas pelo programa não contêm detalhes, circuitos elétricos e comandos de controle dos circuitos. Atualmente está sendo usado também na disciplina o DIALux evo, que possibilita o projeto da iluminação de ambientes internos e externos de forma integrada.

Em função desse antecedente, optou-se por incluir o DIALux na disciplina de iluminação natural na última etapa de análises qualitativas, numa sequência que envolve etapas anteriores da análise de dados medidos em ambientes construídos, do desenho sobre fundo negro e da modelagem física. Para uso no DIALux, os alunos fotografam o ambiente em estudo e reproduzem a cena no programa. A foto e a imagem sintética são comparadas e os resultados obtidos, inclusive os das etapas anteriores, são discutidos. Na etapa seguinte, os alunos elaboram no DIALux modelos a partir de levantamentos de dispositivos de controle da luz do dia (como, por exemplo, no Daylight Design Variations Book, disponível em <sts.bwk.tue.nl/ daylight/varbook/>), para explorar possibilidades de controle e de efeitos da iluminação natural.

Em 2003 foi feita uma análise da usabilidade do Desktop Radiance para uso didático como complemento de um projeto de iniciação científica (MENEGHETTI, 2004). Os resultados do uso na pesquisa foram satisfatórios, mas eles mostraram também a inviabilidade da aplicação didática devido à baixa capacidade de processamento dos computadores pessoais que rodavam o Microsoft Windows na época e à necessidade do domínio do AutoCAD 3D, usado como interface de modelagem pelo programa.

A retomada do uso do Radiance ocorreu em atividades de pesquisa sobre imagens HDR em 2005, mas somente em 2010, através de outra pesquisa de iniciação científica e diante da demanda dos alunos de um meio de representação que permitisse a visualização da iluminação de espaços modelados no SketchUp, passou a ser usado em atividades de ensino.

O SketchUp invadiu paulatinamente as salas de aula das disciplinas do curso de Arquitetura e
Urbanismo da FAAC, à medida que as atualizações davam à interface gráfica mais recursos e facilidades. Os alunos também começaram a se aventurar pelo universo dos renderizadores fotorrealistas, a partir dos modelos criadosno SketchUp. Os renderizadores, porém, não foram criados para simular situações necessariamente reais e mesmo quando se recriam situações próximas a elas, não são conhecidas validações para essa categoria de programas e eles não dispõem de ferramentas de análise.

Aproveitando a preferência dos alunos em relação ao SketchUp, decidiu-se incluir nele ferramentas digitais que possibilitassem a tomada de decisões para seleção de alternativas de projetos arquitetônicos em relação ao aproveitamento da luz natural. O caminho adotado foi o uso de extensões (plugins) que convertem os arquivos gerados pelo SketchUp em arquivos de entrada para o Radiance. Embora a utilização plena do programa envolva um grande tempo de aprendizado, é possível de imediato usar apenas alguns de comandos básicos para gerar arquivos que podem ser transformados em imagens para visualização e análises.

\subsection{O plugin su2rad}

Com a popularização do SketchUp e a distribuição de sua biblioteca para desenvolvimento de extensões, vários plugins passaram a ser criados para expandir os recursos do programa. O su2rad (BLEICHER, 2008) é um plugin do SketchUp cuja finalidade é criar, a partir do arquivo do modelo aberto no programa, um conjunto de arquivos descritores de cena, céu e objetos (materiais, texturas) para serem usados como entrada para a simulação da iluminação através do aplicativo rad, do Radiance. Aparentemente seu desenvolvimento foi interrompido pelo autor em 2010, mas há uma versão operacional disponível em 丸htpp//code.google. complsu2raddownbads/detail?name=su2rad_daysim_103.zips.

A interface do su2rad contém seis abas para configurações:

1. Export: definição do caminho para o qual serão exportados os arquivos, nome da cena do SketchUp a ser usada e opções de qualidade e de dimensões da imagem de saída;

2. Render: definição da posição do observador (interna ou externa ao ambiente), da variável a ser simulada (iluminância ou luminância) e de argumentos usados pelo programa gera- 
dor de imagens do Radiance;

3. Sky: opções de tipo de céu conforme definições da CIE 110-1994 (encoberto, uniforme, intermediário e limpo), data, horário a serem simulados e localização e orientação do modelo;

4. Views: opções de representação de vistas: perspectiva, paralela, angular, cilíndrica, horizontal e estereométrica;

5. Materials: possibilita a substituição de um material definido no SketchUp por um do banco de dados do Radiance.

Para fins de ensino são usadas apenas as definições da aba Export e as básicas das abas Render e Sky, uma vez que os parâmetros de qualidade da imagem, do aplicativo de geração de céu e as propriedades dos materiais não são óbvias aos leigos na utilização do Radiance. Em relação à aba Views, a única representação de vista que produz resultados é a perspectiva.

A partir da versão 8 de uso livre do SketchUp não existe mais a ferramenta de orientação do modelo em relação ao norte; assim, é usado também o plugin location, do mesmo autor do su2rad e disponível em <http://su2rad. googlecode.com/svn/trunk/location/location.rb> para definir a localização e a orientação do modelo.

Inicialmente, a intenção era bastante simples: após elaborar o modelo no SketchUp e exportálo via su2rad, usar o comando de linha rad radiance_input_file.rif (onde radiance_input_file. rif é o arquivo gerado pelo su2rad e rad é o comando do Radiance através do qual aquele arquivo é processado) para gerar uma imagem HDR de distribuição de luminâncias ou de iluminâncias, dependendo do parâmetro de exportação usado no su2rad. A seguir, poderia ser usado o comando winimage imagem sintética.hdr (onde winimage é o programa do Desktop Radiance usado para visualizar imagens HDR, gerar tonemaps, para visualização semelhante à da visão humana, e de escala de falsas cores da variável em estudo - iluminância ou luminância) e criar arquivos de imagens em formatos bitmap, compatíveis com programas de visualização do Microsoft Windows.

Infelizmente, descobriu-se que os alunos dominam muito bem programas com interfaces gráficas, mas têm grandes dificuldades com comandos de linha para navegar por pastas e executar comandos.

\subsection{Desenvolvimento de interfaces gráfi- cas para executar scripts do Radiance}

Para contornar a dificuldade acima exposta foi criado um programa, o MinRad, uma interface mínima, cujo objetivo era simplesmente executar as linhas de comando mencionadas anteriormente ao clicar de um botão, ao estilo Microsoft Windows (Figura 1). O uso do MinRad era muito simples: bastava localizar o arquivo .rif gerado pelo su2rad e clicar no botão <Processar> para gerar a imagem HDR; o caminho do arquivo gerado era já colocado no segundo campo de edição e, clicando-se no botão <Abrir>, o winimage era executado, abrindo a imagem para pós-processamento. Em 2011 o MinRad foi ligeiramente incrementado, através da inclusão da opção do tratamento pelo aplicativo evalglare da imagem gerada pelo Radiance (Figura 2), com funcionamento similar à versão anterior. Foi escolhido como versão do Radiance o Desktop Radiance, por conter aplicativos para o ambiente Microsoft Windows como o winimage, já comentado e o falsecolor, para a criação de imagens de escalas de falsas cores.

Figura 1: Tela do MinRad.

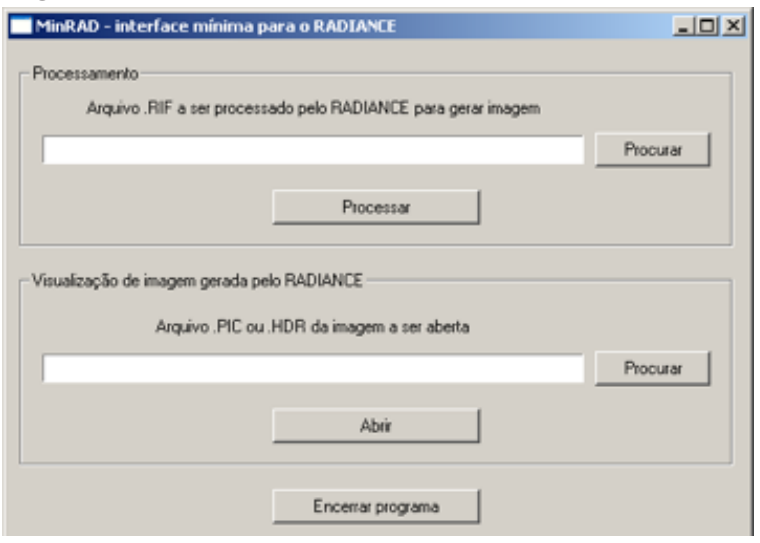

Figura 2: Tela da nova versão do MinRad, com a adição da opção de tratamento da imagem pelo evalglare.

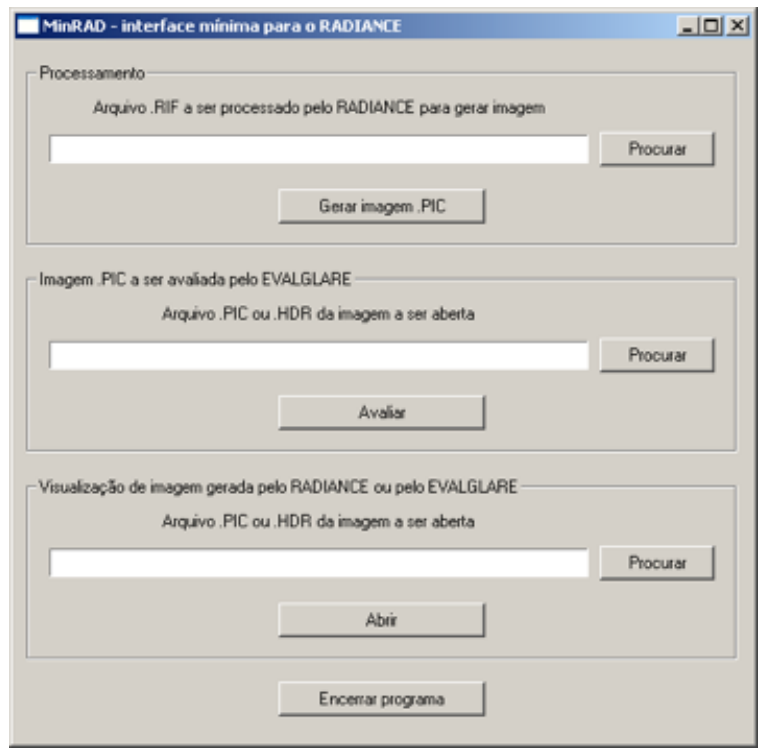


O programa evalglare (WIENOLD; REETZ; KUHN, 2004) cria uma imagem em tons de cinza a partir da imagem HDR original e, sobre ela, áreas coloridas correspondentes às superfícies potencialmente causadoras de ofuscamento; além disso, apresenta, na tela ou em um arquivo, valores de 5 índices de ofuscamento e da luminância veladora. Infelizmente os resultados quantitativos não podiam ser contabilizados a partir do processamento realizado através do MinRad, uma vez que eles deveriam ser calculados pelo evalglare a partir de imagens em projeção angular, opção não suportada diretamente pelo SketchUp e inoperante no su2rad. Assim, as manchas coloridas sobre as imagens indicavam apenas a necessidade de revisão do projeto.

Em 2012 optou-se por fazer uma alteração drástica na interface, que passou a ser chamada SimulaLuz (Figura 3). Nessa versão era possível salvar uma única cena a partir do SketchUp e simular um número ilimitado de datas e horários, sob até 6 tipos diferentes de céu (cf. CIE 1101994, padrões do Radiance): limpo com e sem sol, intermediário com e sem sol, encoberto e uniforme. Poderiam ser geradas simultaneamente imagens de luminância nas representações de tonemap, escala de falsas cores e potencial de ofuscamento a partir do observador (em representação angular e com os respectivos valores de índices de ofuscamento) e imagens de planta da sala simulada em escala de isoiluminâncias sobre planos horizontais. O SimulaLuz manipulava os arquivos de saída do su2rad de forma a alterar as datas e os horários, as características do céu, os parâmetros de qualidade das imagens, o tipo de imagem de saída e o tipo de projeção dessas imagens para representar o potencial de ofuscamento e a distribuição de iluminâncias sobre planos horizontais. As imagens finais já eram geradas no formato bitmap (.bmp), facilitando sua visualização e uso em relatórios. Além disso, a interface passou a ter um menu a partir do qual era possível gravar e recuperar posteriormente os dados de uma simulação e um arquivo de ajuda, com a descrição da interface e da teoria envolvida (p. ex. sobre índices de ofuscamento) e um tutorial para a operação do programa.

\subsection{Resultados do uso dos programas}

A Figura 4 ilustra um resultado da simulação feita no DIALux de um espaço bastante conhecido pelos alunos, seu próprio dormitório.

Esse exercício é a introdução à modelagem digital para simulação da iluminação, onde o
Figura 3: Tela do SimulaLuz.

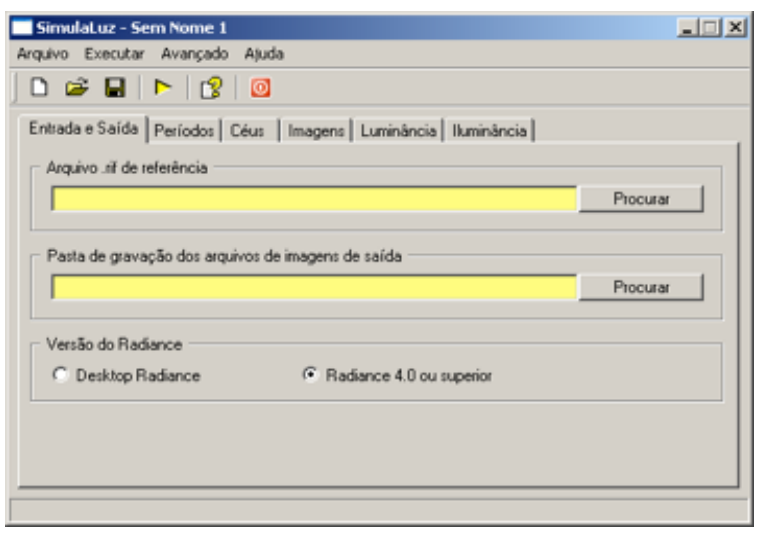

Figura 4: Imagens usadas na análise qualitativa: a) Foto do objeto de estudo; b) Imagem do objeto gerada pelo DIALux sob céu limpo com sol; c) Imagem do objeto gerada pelo DIALux sob céu encoberto
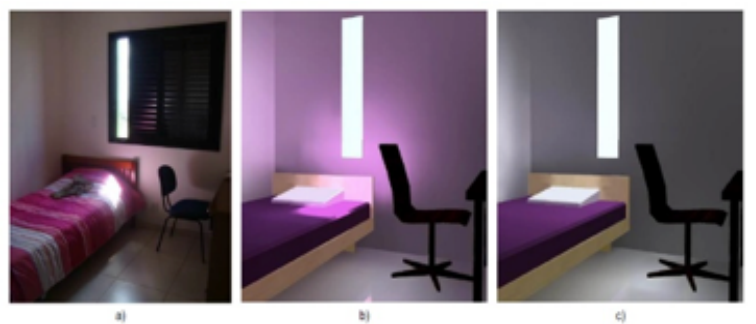

aluno é colocado frente às possibilidades e limitações do modelador. Verificam-se, na imagem simulada, a simplificação do modelo e o uso de elementos da biblioteca de mobiliário do DIALux que têm a mesma função, embora não exatamente a mesma forma. A partir desse exercício, discute-se a influência de parâmetros do modelo na difusão da luz do dia, como espessuras de paredes, coeficientes de reflexão e grau de polimento das superfícies.

A Figura 5 mostra estudos de controle da luz do dia elaborados a partir de um levantamento bibliográfico. Como exemplificado nas imagens, os modelos elaborados no DIALux tenderam a ser ortogonais, enquanto no SketchUp os alunos exploraram melhor as possibilidades formais derivadas das facilidades de modelagem que este último programa apresenta.

A exploração do potencial do SketchUp para a rápida elaboração de modelos envolvia também um exercício no qual os alunos deveriam propor soluções para melhorar o aproveitamento de uma sala de aula do câmpus da UNESP em Bauru sujeita a períodos de exposição à radiação solar direta e a períodos de aproveitamento insuficiente de iluminação natural. As soluções propostas deveriam ser simuladas com o auxílio do Radiance e os resultados analisados em termos de distribuição de iluminâncias no plano 
Figura 5: Estudos de controle da luz do dia executados: a) no DIALux e b) no Radiance.

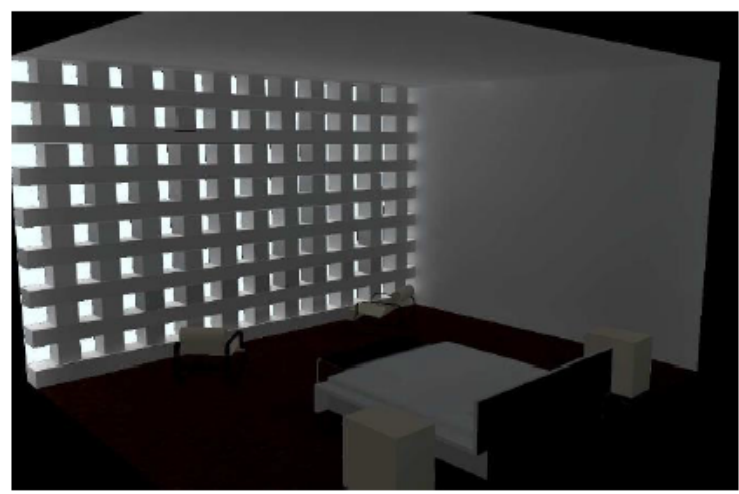

a)

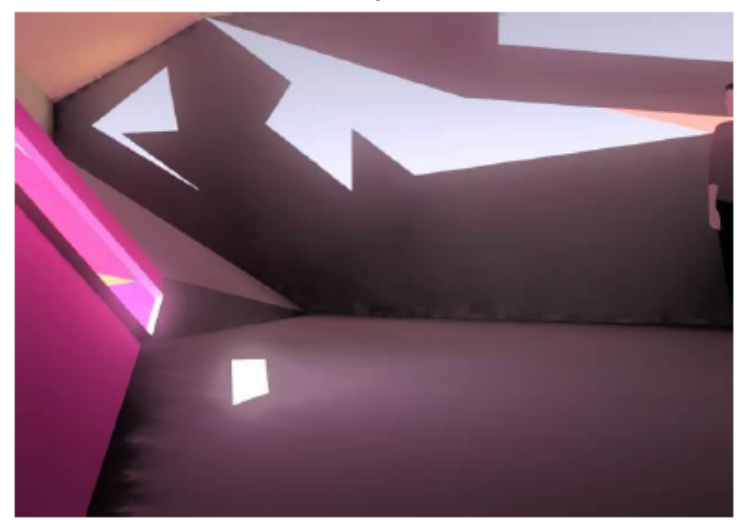

b)

das mesas e de avaliação do potencial de ofuscamento. No primeiro caso havia as restrições do atendimento do valor mínimo segundo a antiga NBR 5413 - lluminância de Interiores (500 Ix sobre os planos das mesas) e da não extrapolação de aproximadamente 2000 Ix, o que, na prática, ocorre quando há incidência de radiação solar direta. O potencial de ofuscamento deveria ser avaliado visualmente, pela ocorrência de manchas coloridas nas imagens, e pelo valor máximo para salas de aula do UGR (Unified Glare Rating - índice de ofuscamento unificado), definido pela NBR ISO/ CIE 8995-1 - "lluminação de ambientes de trabalho. Parte 1: interior" como 19 (ASSOCIAÇÃO BRASILEIRA DE NORMAS TÉCNICAS, 2013).

Para o desenvolvimento do exercício era fornecido o modelo digital da sala de aulas. As soluções propostas deveriam melhorar o aproveitamento da luz natural em uma sala de aulas, evitando a radiação solar direta e maximizando o ganho de luz ao longo do dia; além disso, deveria se mantida a cobertura em 2 águas com ático e a dimensão lateral das janelas deveria acompanhar a modulação dos painéis que compõem as paredes.

A Figura 6 mostra a proposta de um aluno de 2012 para o exercício. Nela verifica-se a inclusão de brises / prateleiras de luz na janela a norte, rebaixamento e ampliação do beiral na fachada sul e inclusão de uma abertura zenital. A eficiência da solução em relação ao sombreamento pôde ser verificada no próprio SketchUp, através da ferramenta de configuração de sombra.

Figura 6: Corte transversal do modelo digital da sala de aula: a) Original; b) Proposta de intervenção.

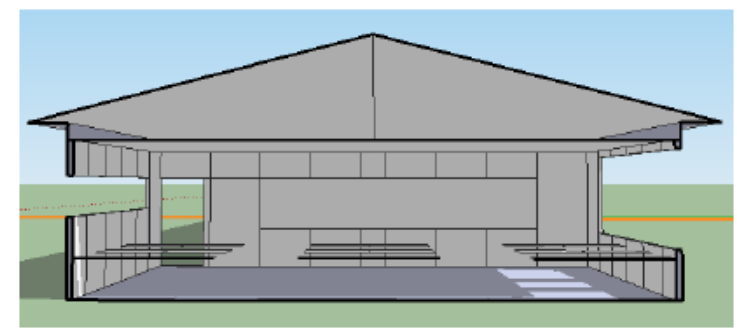

a)

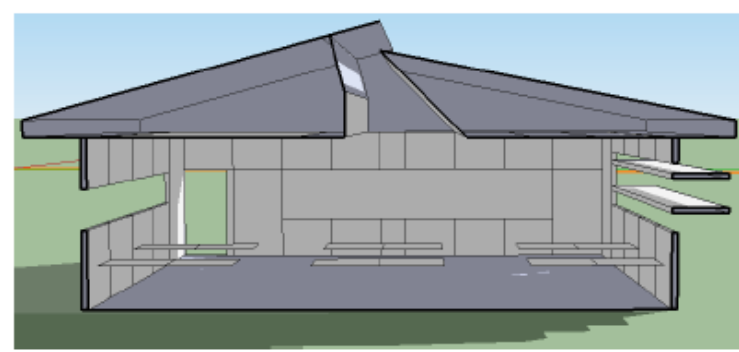

b)

Depois de resolvido o sombreamento, o modelo da proposta era exportado via su2rad e simulado no Radiance através da interface SimulaLuz. Eram solicitadas simulações às 9, 11, 13 e 17 horas dos solstícios de verão e inverno e equinócios, sob céus totalmente limpo com sol e encoberto, o que representavam 48 imagens de saída. Algumas imagens resultantes dessas simulações são mostradas na Figura 7. Observase que, para o horário simulado, a proposta cria algumas áreas de ofuscamento, pela reflexão da luz na abertura zenital e na janela norte, devido à redução da luminância das superfícies adjacentes. No entanto, a iluminância ficou melhor distribuída e dentro dos limites determinados no enunciado.

\subsection{Discussão dos resultados do uso de programas de simulação}

A simulação computacional da iluminação veio completar o que as ferramentas de estimativa de quantidade de luz do dia não forneciam, a visualização do modelo iluminado pela luz do dia. Esse aspecto, aliado ao uso de interfaces gráficas amigáveis, tornou os programas de simulação bem-vindos aos alunos.

O fato de iniciar as simulações com o DIALux, 
Figura 7: Imagens do gerada pelo evalglare para um observador posicionado no fundo da sala às $11 \mathrm{~h}$ do dia 21 de junho sob céu limpo com sol: c) Original; d) Proposta. Distribuição de iluminâncias nos planos horizontais das mesas e do piso às 11 h do dia 21 de junho sob céu limpo com sol: e) Original; f) Proposta.

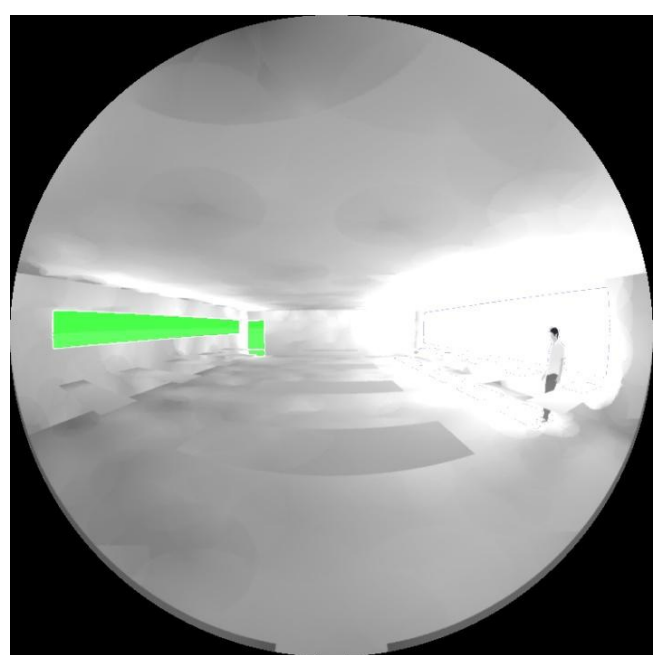

C)

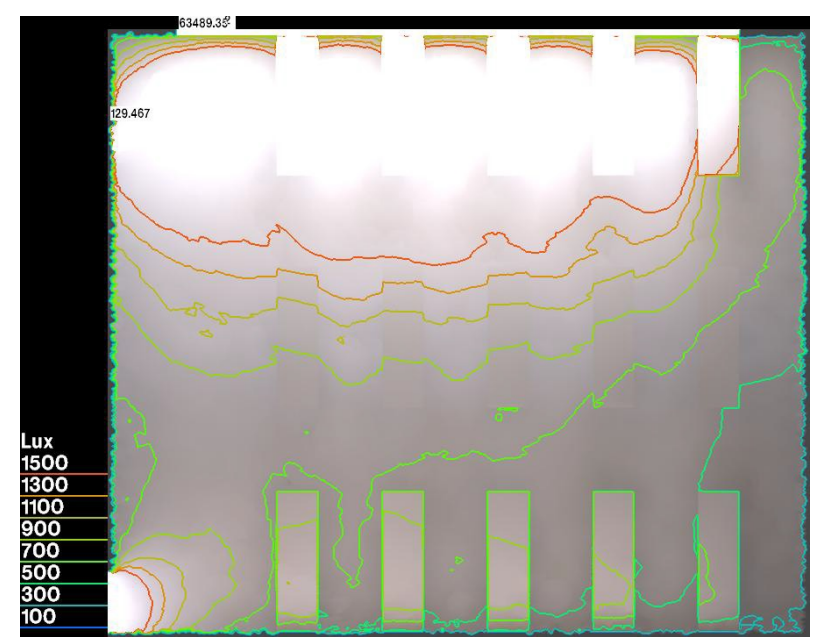

e)

um programa cuja interface gráfica é mais restritiva em termos de possibilidades geométricas requer atenção do aluno de um lado para simplificar o modelo ao básico para fornecer os resultados da iluminação, e não para obter uma imagem fotorrealista. Por outro lado, essa limitação também o incentiva a usar a criatividade para contorná-la, o que na maior parte das vezes é bem sucedido.

Ao final da sequência de exercícios de simulação como SketchUp+Radiance era solicitado que alunos comentassem livremente sobre as dificuldades encontradas das técnicas de modelagem computacional, no aprendizado especifico da simulação através do SketchUp+Radiance, no desenvolvimento do projeto da sala de aula e na análise de resultados. Os comentários mais comuns sobre a primeira

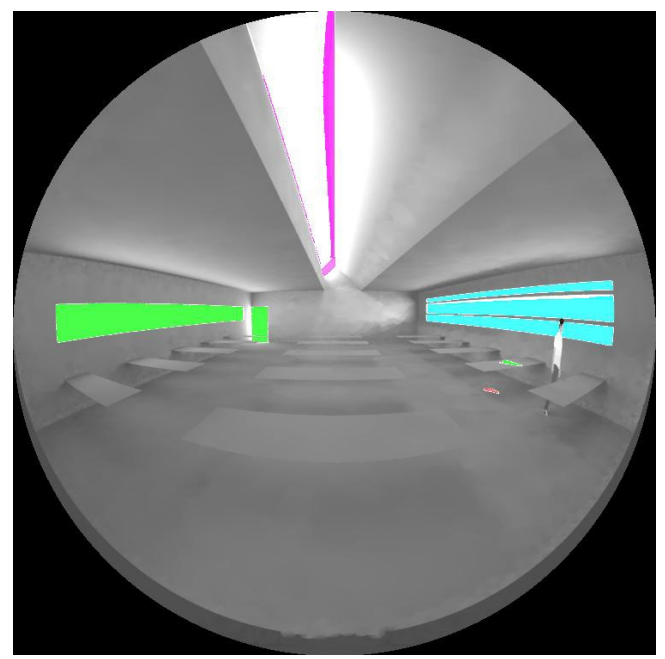

d)

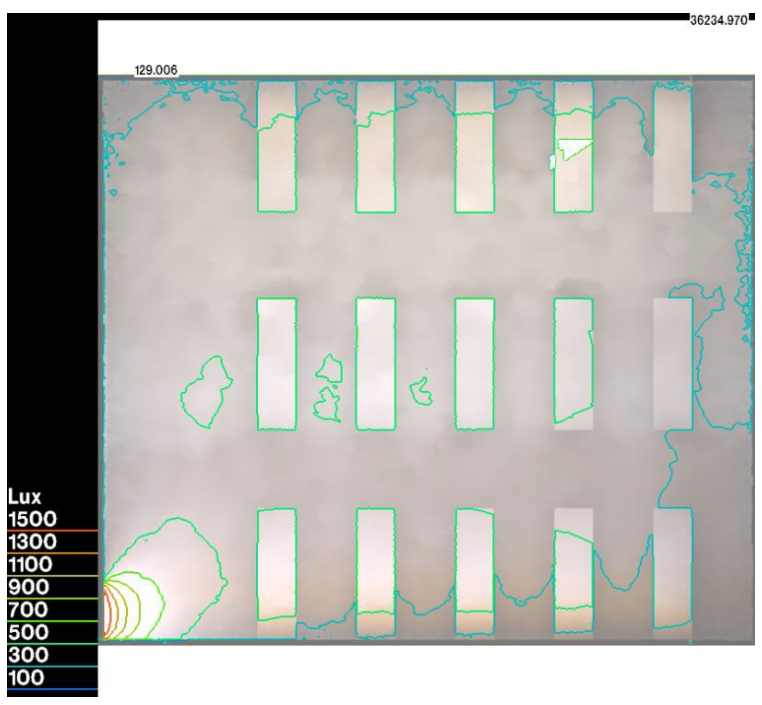

f)

questão foram sobre a diferença de enfoque entre a simulação da iluminação e a renderização fotorrealística; sobre a segunda, mais relacionadas a questões técnicas, como erros ocorridos durante a exportação a partir do SketchUp e na execução do Radiance. O desenvolvimento do projeto e a análise dos resultados não apresentaram problemas, segundo a maioria dos alunos: a primeira, feita no SketchUp era relativamente simples, embora trabalhosa. Finalmente, segundo os alunos, a etapa de análise dos resultados era bastante facilitada pelos resultados em forma de imagens.

O uso do su2rad e do Radiance através do SimulaLuz apontaram algumas dificuldades dos alunos, não no manuseio desses recursos, mas, o que não era esperado, na modelagem via SketchUp. Enquanto o modelo fica no próprio 
SketchUp, o programa tolera uma série de problemas conceituais em modelagem, sendo o mais comum a intersecção de superfícies. Esse problema aparece com frequência em blocos importados de mobiliário, além de superfícies do próprio modelo em execução, quando se trabalha com grupos ou componentes. Outro problema é a descontinuidade de superfícies, causada pelo apagamento de alguma delas, localizada em geral em um local pouco visível. O primeiro tipo de problema causa a falha na conversão de arquivos pelo su2rad, enquanto a outra leva o Radiance à geração de resultados inconsistentes.

Devido à concepção para ambientes Unix do Radiance, suas pastas de trabalho, assim como seus arquivos, não podem ter caracteres que não os alfanuméricos, sem acentos e espaços, o que se revelou uma fonte de erros constante na operação do SketchUp+Radiance.

O fato dos alunos terem que sair do SketchUp e usar a interface SimulaLuz para realizar as simulações revelou dificuldades básicas: de navegação pelas pastas do disco para localizar os arquivos necessários ao segundo programa; em executar um programa de forma sistemática, abrindo-o para carregar 0 arquivo a ser executado, ao invés de simplesmente clicar duplamente nele, já que não foi feita nenhuma associação dos arquivos .rif ao SimulaLuz; ao se confundir com uma série de arquivos de mesmo nome mas com extensões diferentes, uma vez que por padrão o Microsoft Windows oculta as extensões conhecidas.

No início de 2012 os computadores da sala de Recursos Computacionais em Arquitetura e Urbanismo, onde são ministradas as disciplinas de iluminação, foram atualizados, o sistema operacional passou do Microsoft Windows XP para o Microsoft Windows 7 e a geração de imagens de iluminância deixou de funcionar no Desktop Radiance. O problema foi resolvido atualizando-se o Radiance através da versão compilada para ambiente Microsoft Windows disponível no sítio do NREL OpenStudio, em $<$ https://openstudio.nrel.gov/getting-starteddeveloper/getting-started-radiance>.

Finalmente, poucos alunos fazem análises a partir dos valores de índices de ofuscamento gravados em arquivos texto com os mesmos nomes das imagens, o que reforça a referência feita anteriormente à necessidade de transformar os resultados em desenhos associados às imagens (a exemplo dos resultados de probabilidade de ocorrência de ofuscamento do programa DAYSIM).

\section{Conclusões}

O processo de inclusão digital do ensino de iluminação natural do curso de Arquitetura e Urbanismo da FAAC-UNESP está consolidado e pode ser considerado bem sucedido: os alunos passam a compreender melhor os parâmetros envolvidos na interação da luz do dia com o ambiente construído e, dessa forma, a melhor projetar as aberturas e outros elementos arquitetônicos para conseguirem os efeitos visuais desejados.

As ferramentas empregadas possuem interfaces gráficas de modelagem de fácil aprendizado, quando não já de conhecimento dos alunos, em se tratando do SketchUp. A situação ideal seria se as interfaces de modelagem usadas nessa etapa do projeto permitissem a evolução do projeto em sistemas de CAD, a exemplo do Autodesk Ecotect (o qual, aliás, tem uma interface para simular a iluminação via Radiance), mas o DIALux não dispõe desse recurso e ele foi suprimido da versão gratuita do SketchUp a partir da versão 7.

Provavelmente uma série de problemas relatados em relação à navegação e gerenciamento de arquivos não existiria se a interface SimulaLuz fosse integrada ao SketchUp, como o é o su2rad. Por outro lado, se a execução do su2rad é rápida, uma vez que se trata somente de uma conversão de arquivos executar o SimulaLuz significa executar uma sequência de comandos do Radiance, o que, dependendo da quantidade de variáveis simuladas, demanda um longo tempo, no qual se ficaria impossibilitado de trabalhar com o SketchUp. Nesse caso, acredita-se que seja conveniente manter o SimulaLuz independente do SketchUp.

O SimulaLuz continua evoluindo. Atualmente, conta com o recurso de adicionar, numa mesma cena exportada do SketchUp, vários observadores, para simular índices de ofuscamento a partir de diversos pontos do ambiente simulado. Também pode usar, ao invés de céus padrão CIE, distribuições de luminosidade baseadas nas irradiâncias direta e difusa no plano horizontal, extraídas de um arquivo climático no formato epw (Energy Plus Weather).

Outros recursos ainda podem ser incluídos no SimulaLuz, como o cálculo de índices de autonomia de luz do dia, a exemplo do DAYSIM. No entanto, essa inclusão progressiva de recursos para melhor explorar o potencial do Radiance deve ser feita com cautela e avaliada passo a passo, tendo em vista a finalidade antes 
de tudo didática da interface.

\section{Referências}

ASSOCIAÇÃO BRASILEIRA DE NORMAS TÉCNICAS. NBR 15215-3: Iluminação natural, parte 3: procedimento de cálculo para a determinação da iluminação natural em ambientes internos. Rio de Janeiro, 2005, Versão Corrigida: 2007.

ASSOCIAÇÃO BRASILEIRA DE NORMAS TÉCNICAS. NBR ISO/CIE 8995-1: Iluminação em ambientes de trabalho. Parte 1: interior. Rio de Janeiro, 2013.

ATANASIO, V.; PEREIRA, F. O. R.; PEREIRA, A. T. C. Experimental laboratory for teaching architecture lighting in a virtual learning environment. In: PLEA2006 - The 23 rd Conference on Passive and Low Energy Architecture, Geneva, Switzerland, 6-8 September 2006. Proceedings... Geneva: Université de Genève, PLEA, v. 2, p. 761-766, 2006.

BLEICHER, T. su2rad - Radiance exporter and other Ruby scripts for Google Sketchup - Google Project Hosting. 2009. Disponível em: <http://code.google. com/p/su2rad/>. Acesso em: 23 jul. 2010.

CABÚS, R. TROPLUX 5: guia do usuário. Maceió: Instituto Lumeeiro, 2011.

CLARO, A.; PEREIRA, F.O.R.; LEDO, R. Z . APOLUX - An Innovative Computer Code for Daylight Design and Analysis in Architecture and Urbanism. In: 9th International IBPSA Conference, 2005, Montreal. Proceedings.... Montreal : IBPSA, 2005. v. único. p. 199-206.

COMPAGNON, R. The RADIANCE simulation software in the architecture teaching context. In: Florence International Conference for Teachers of Architecture, 2, 1997. Proceedings.... Firenze: Alinea Editrice, 1997. Disponível em: <http://radsite.lbl.gov/ radiance/papers/rctia97/rct97.htm>. Acesso em: 2 jul. 2005.

INANICI, M. N. Transformation of high dynamic images into virtual lighting laboratories. In: International IBPSA Conference, 8, 2003, Eindhoven, Netherlands. Proceedings.... Eindhoven, Netherlands: IBPSA, 2003, p. 539-546.

LEE, T. -S.; HARDIN, M. Daylight visual effects: an interactive multimedia courseware prototype for beginning design students. ACM SIGGRAPH Computer Graphics - special issue: focus: computer graphics education, v. 30, n. 2, p. 51-53, 1996.

MAGAGNIN, R. C.; BARON, C. M. P.; HIRAO, H.; SALCEDO, R. F. B.; AMARAL, C. S. Diretrizes para os cursos de graduação da Unesp: Arquitetura e Urbanismo : estudos resultantes do processo de articulação e integração dos cursos de Arquitetura e Urbanismo da Unesp. São Paulo: UNESP-PROGRAD, 2012. Disponível em: <http://unesp.br/prograd// mostra_arq_multi.php?arquivo=9621>. Acesso em: 20 fev. 2013.

MARDALJEVIC, J. Daylight simulation: validation, sky models and daylight coefficients. PhD. Thesis, De Montfort University, Leicester, UK, 1999.

MENEGHETTI, L. H. B. lluminação natural em edificação universitária: avaliação e aplicação de um software. Relatório final de bolsa de Iniciação Científica FAPESP. Bauru, 2004.

PEREIRA, F. O. R.; CASTAÑO, A. G.; ATANASIO, V. Teaching and learning the daylighting phenomenon in architecture with physical models. In: International Conference on Passive and Low Energy Architecture, 24th., 2007, Cingapura. Proceedings.... Cingapura: Research Publishing Services, 2007. p. 706-711.

ROBERTS, A.; MARSH, A. ECOTECT: Environmental prediction in architectural education. In: 19th ECAADE - Education for Computer Aided Architectural Design in Europe, Helsinki, Finland, 2001. Proceedings... Helsinki, Finland, p. 342-347.

SABRY, H.; SHERIF, A.; FEKRY, A; RAKHA, T. Integration of daylighting simulation software in architectural education. In: Computing in Civil and Building Engineering, Nottingham, UK, 2010. Proceedings of the International Conference. Nottingham, UK: Nottingham University Press, p. 597305.

SCARAZZATO, P. S.; MONTEIRO, A. M. R. de G.; MARTAU, B. T.; SILVA, F. D. da . Proposta de uma metodologia de ensino de iluminação para cursos de graduação em arquitetura. In: ENCAC-ELACAC 2005 - VIII Encontro Nacional sobre Conforto no Ambiente Construído/ IV Encontro Latino-Americano sobre Conforto no Ambiente Construído, 2005, Maceió, Alagoas. Anais... Maceió: ANTAC, 2005. (CD-ROM).

SHIKDER, S. Evaluation of four artificial lighting simulation tools with virtual building reference. In: 2009 Summer Computer Simulation Conference, Istanbul, Turkey. SCSC '09 Proceedings. Visa, CA: The Society for Modeling and Simulation International, 2009. p. 430-437.

REINHART, C. F. Daylight availability and manual lighting control in office buildings - simulation studies 
and analysis of measurements. Ph.D. thesis, Technical University of Karlsruhe, Faculty of Architecture, Germany, 2001.

WARD, G. J. The RADIANCE Lighting Simulation and Rendering System, In: SIGGRAPH 94 Annual Conference, Orlando, Florida, 1994. Proceedings of '94 SIGGRAPH conference. Orlando: ACM SIGGRAPH, p. 459-72, 1994.

WIENOLD, J.; REETZ, C.; KUHN, T. Evalglare: a new RADIANCE-based tool to evaluate glare in office spaces. 3rd International RADIANCE Workshop, Fribourg, 11-12 october 2004. CD Online, Fribourg: Ecole d'Ingénieurs et d'Architectes, 2004. Disponível em: <http://www.radiance-online.org/radianceworkshop3/cd/>. Acesso em: 20 out. 2005. 